\section{TISSUE-RESIDENT NATURAL KILLER CELLS RESEMBLING INTRAEPITHELIAL ILC1 HAVE POTENT ANTI-TUMOR ACTIVITY IN HUMAN HEAD AND NECK CANCER AND REPRESENT A NOVEL CLASS OF EFFECTOR CELLS FOR IMMUNOTHERAPY}

June Shin*, Nina Horowitz, Quan Tran, Chen Chen, Uriel Moreno-Nieves, Joshua Tay, Saumyaa Saumyaa, John Sunwoo*. Stanford University, Stanford, CA, USA

Background Natural killer (NK) cells comprise a subset of the innate lymphoid cell (ILC) family. Although NK cells have been observed to be present in most solid tumors, their role in the protection against tumor formation in humans has been unclear. Studies have been hampered by the heterogeneity of NK cells within the tumor microenvironment (TME) and lack of information about the broader ILC subsets found in tumors. Further, there is an increasing recognition of plasticity between NK cells and other ILC family members in various disease contexts, calling for a broader examination of ILCs within solid tumors. We previously analyzed the ILC population in primary samples from human head and neck squamous cell carcinoma (HNSCC) and matched blood by single-cell RNA sequencing (scRNA-seq). ${ }^{1}$ Those studies revealed that peripheral NK cells differentiate along two divergent trajectories in the TME, resulting in different end-states: one with a hyporesponsive phenotype and another possessing potent antitumor activity and resembling intraepithelial ILC1s (ieILC1s).

Methods In vitro co-culture approaches and in vivo mouse models were used to investigate the ability of peripheral NK cells to differentiate into alternate ILC states with heterogeneous functions. Cytotoxicity assays were used to assess functional activity of in vitro derived ieILC1-like cells. Adoptive cell transfer of ieILC1-like cells into tumor-bearing mice was also used to assess anti-tumor function.

Results Peripheral human NK cells could be efficiently differentiated into ieILC1-like cells using an in vitro co-culture system. These ieILC1-like cells had enhanced natural cytotoxicity against target cells compared to conventional IL-15-activated and K562-expanded NK cells. In addition, they infiltrated the TME efficiently and were a more effective means of adoptive cell therapy against HNSCC solid tumor xenografts in vivo compared to conventional NK cells.

Conclusions Our data indicate that peripheral NK cells change cell states within the TME of HNSCC. The heterogeneity in the relative proportion of the cell states may influence host response to tumors. We identified the ieILC1-like cell state to be the phenotype with the most potent anti-tumor activity within the TME. Importantly, this cell state can be induced from peripheral donor NK cells ex vivo for differentiation into and expansion of highly active ieILC1-like cells, providing a platform for a novel class of effector cells for adoptive cell immunotherapy.

Acknowledgements These studies were supported by the Lokey Stem Cell Research Building (SIM1) Flow Cytometry core facility for cell sorting and flow cytometric analysis and the Stanford Cancer Institute Tissue Bank for procurement of tumor samples and blood. This work was supported by funding from the National Institutes of Health (R01CA158516; R35DE030054; U54CA209971) to J.B.S.

\section{REFERENCE}

1.. Moreno-Nieves UY, Tay JK, Saumyaa $S$, Shin JH, Horowitz NB, Mohammad IA, Luca B, Mundy DC, Gulati GS, Bedi N, Chang S, Chen C, Kaplan MJ, Rosenthal $E L$, Holsinger FC, Divi V, Baik FM, Sirjani DB, Gentles AJ, Newman AM, Freud AG, Sunwoo JB. Landscape of ILCs in human head and neck cancer reveals divergent NK cell states in the tumor microenvironment. Proc Natl Acad Sci U S A 2021:118(28):e2101169118

Ethics Approval The studies reported here were approved by the Stanford Institutional Review Board (IRB 11402) and the Stanford Administrative Panel on Laboratory Animal Care (APLAC 20547).

http://dx.doi.org/10.1136/jitc-2021-SITC2021.215 